discernment of a possible method of controlling sex.

It is interesting to note that pursuit of this speculation leads one to a view of the paramount importance of the individual as a whole in determining its characters, and in this respect recalls Darwin's hypothesis of pangenesis. It may be pointed out, moreover, that quite apart from theoretical questions the conditions which determine whether an animal is to be hermaphrodite or bisexual must be considered before any reasonable hope can be entertained of understanding the processes underlying sexual differentiation in a bisexual species. Indeed, some inkling of the need for sex might be obtained from an understanding of the conditions resulting in hermaphroditism, and an explanation of sex on purely physical lines would be captivating from the point of view of the student of nature as a whole. The old idea that an individual is female because its metabolism is mainly anabolic, or male because mainly katabolic, is unsatisfactory, and it has already been pointed out that it is just as likely that an individual is anabolic because of its femaleness, or katabolic because of its maleness.

In reviewing the incidence of hermaphroditism and bisexuality throughout the animal kingdom (again excluding Protozoa) one cannot but be impressed by the independence shown by the organism, which would appear to be able to order its sexual manifestations in a manner entirely independent of phylogenetic considerations, and suggests such a ready response to conditions of life as is perhaps not generally conceived.

The practical points arising out of these speculations may be summarised as follows: Is there a fundamental physical difference between sedentary and active organisms in the whole body or in parts, and if so, is this difference correlated in any way with particular sexual manifestations? To what degree is our present knowledge of the sexual conditions in slowmoving or fixed animals reliable? On the basis of the speculations advanced here any animals which are sedentary, fixed, or slow-moving may be suspected of hermaphroditism in some form.

No wholly satisfactory explanations have been advanced for either hermaphroditism, bisexuality, or indeed sex itself. Sex is universally expressed amongst the higher living organisms at least, so that one is tempted to ask: Is sex merely a property of living matter and hermaphroditism that modification of the property resultant upon the absence of motion in the living organism, and bisexuality that modification dependent upon free motion? For there appear to be sufficient grounds for rejecting the idea that animals are sedentary because they are hermaphrodite.

J. H. ORTON.

The Laboratory, Plymouth, September I9.

\section{The Separation of Mercury into Isotopes.}

EARLY in I920 Harkins and Broeker reported a separation of chlorine into isotopes, which amounted at that time to an increase in density equal to $I_{55}$ parts per million. About six months later Brønsted and von Hevesy reported a separation of mercury, which was, however, only about one-thirtieth as great, or 50 parts per million. On account of the slightness of the density change reported for mercury, the evidence that it had been separated did not appear to be conclusive, so it seemed worth while to attempt a confirmation by the same methodthat is, a vaporisation at low pressures. As a result of 4 cuts of 2 on the heavy fraction or residue the density has been increased by 69 parts per million, and by the same number of cuts on the light fraction it has been decreased 62 parts, or the total density change obtained is 133 parts per million, or 0.027 units of atomic weight.

The mercury was purified by electrolysis, by five fractional distillations at low pressure in a current of pure air, and by one fractional distillation in a high vacuum.

The present evidence that an actual separation of isotopes has been obtained with mercury rests largely in the quantitative agreement between the results of Brønsted and von Hevesy and ourselves with respect to the extent of the separation obtained by a definite cut, which will be termed the efficiency of the process. If the efficiency of our ideal apparatus, in which solid carbon dioxide and ether were used for cooling, is rated as roo per cent., then the efficiency attained by the previous investigators is 75 per cent., while our less ideal apparatus gave 93 per cent. when operated slowly and as low as 80 per cent. when operated rapidiy.

We have obtained evidence which seems to indicate that a slight separation of the isotopes of mercury has been secured by a very slow distillation at very low pressures, though a more rapid distillation gave no detectable difference in density.

The relative changes produced in the atomic weights of different elements by a definite cut may be termed the separation coefficients. These have the values listed below as determined by calculation, the coefficients for chlorine compounds representing the change in the atomic weight of chlorine. The calculated coefficients are :-Neon, o.00843; magnesium, 0.00868 ; lithium, 0.00450; nickel, 0.00758 ; hydrogen chloride, 0.00950 ; methyl chloride, 0.0069o; chlorine, 0.00494 ; methylene chloride, 0.004I3; chloroform, 0.00295 ; carbon tetrachloride, 0.00229 ; and hydrogen bromide, 0.00614 . Thus the atomic weight changes most rapidly when chlorine is used in the form of hydrogen chloride. The experimentally determined coefficient for mercury is 0.00570 , which is not specially large.

The rate of separation of two isotopes is very nearly proportional to the square of the difference of their atomic weight (or molecular weight when a compound is used), to the product of the mol fractions of the two isotopes, to the logarithm of the cut, and inversely proportional to the atomic (or molecular) weight. This statement and the above coefficients apply as well to molecular diffusion at low pressures as to vaporisation at low pressures.

It is of interest to note that many molecular substances must appear in many isotopic forms. Thus if there are two isotopes of chlorine and three of magnesium there are nine isotopic forms of $\mathrm{MgCl}_{2}$ and seven forms of $\mathrm{C}_{6} \mathrm{Cl}_{6}$, while if there are six isotopes of mercury there are sixty-three isotopic forms of $\mathrm{Hg}_{2} \mathrm{Cl}_{2}$, which occurs in the form of a vapour. Five of the seven isotopic hexachlorobenzenes also occur in several isomeric forms. Isotopes may be able to produce stereoisomerism with respect to infra-red rays.

Wrlliam D. Harkins.

R. S. Mulliken.

University of Chicago, August $3 \mathrm{I}$.

\section{Relation of the Hydrogen-ion Concentration of the Soil to Plant Distribution.}

THIS subject has recently assumed prominence among ecologists and soil chemists, and Dr. Atkins's interesting letter, with its valuable data, published in NATURE for September I5 (p. 80), directs general attention to it and to the need and scope for further work thereon.

It seems very desirable to bear in mind that there are strong indications that the relation between the 